\title{
The Raman spectrum of grossular garnet. A quantum mechanical simulation of frequencies and intensities
}

\author{
Lorenzo Maschio, ${ }^{1}$ Raffaella Demichelis, ${ }^{2}$ Roberto Orlando, ${ }^{1}$ \\ Marco De La Pierre, ${ }^{1}$ Agnes Mahmoud, ${ }^{1}$ and Roberto Dovesi ${ }^{1}$ \\ ${ }^{1}$ Dipartimento di Chimica, Università degli Studi di Torino and \\ NIS (Nanostructured Interfaces and Surfaces) Centre of Excellence, \\ Via P. Giuria 7, 10125 Torino, Italy \\ ${ }^{2}$ Nanochemistry Research Institute, Department of Chemistry, \\ Curtin University, GPO Box U1987, Perth WA 6845, Australia
}

(Dated: February 17, 2014)

\begin{abstract}
Raman spectroscopy is a standard and powerful investigation technique for minerals, and garnet is one of the most observed and visible minerals, undoubtfully important both as a witness of our planet's evolution, and as a main component in many high-tech applications. This paper presents the Raman spectrum of grossular, the calcium-aluminium end-member of garnets $\left(\mathrm{Ca}_{3} \mathrm{Al}_{2} \mathrm{Si}_{3} \mathrm{O}_{12}\right)$, as computed by using an ab initio quantum-mechanical approach, an all-electron Gaussian-type basis set and the hybrid B3LYP functional. The frequencies of the 25 Raman active modes are in excellent agreement with the available experimental measurements, with the mean absolute difference being between 5 and $8 \mathrm{~cm}^{-1}$. The apparent disagreement between a few experimental $v s$ calculated data can be easily justified through the analysis of the corresponding calculated peak intensities, which is very low in all of these cases. The intensities of the Raman active modes of grossular were calculated here for the first time, thanks to a recent implementation by some of the present authors that allows for accurate predictions of the Raman spectra of minerals. To the authors' knowledge, there are no tabulated data sets for Raman intensities of grossular, though qualitative information can be extracted from the published spectra. This study can then be considered as an accurate reference data set for grossular, other than a clear evidence that quantum-mechanical simulation is an actual tool to predict spectroscopic properties of minerals.
\end{abstract}

Keywords: grossular, Raman intensities, wavenumbers, ab initio calculation, CRYSTAL code 


\section{INTRODUCTION}

The crucial role of garnets in our everyday's life and in our planet history has been recently highlighted by all the main world Geological, Mineralogical and Geochemical associations through the publication of a themed issue on the journal Elements (vol. 9, issue 6, 2013). In this context, achieving a comprehensive knowledge of their crystal structure and properties as a function of composition at the atomic level is expected to help in the interpretation of the experiments and to provide accurate reference data sets that allow for making realistic predictions in the design of new garnet-based devices. ${ }^{1,2}$

The first purpose of this paper is improving the current knowledge of the Raman vibrational spectrum of grossular, one of the main end-members of the garnet family having ideal formula $\mathrm{Ca}_{3} \mathrm{Al}_{2} \mathrm{Si}_{3} \mathrm{O}_{12}$. In the last two decades, a number of experimental Raman studies on grossular has been published, ${ }^{3-8}$ one of which reporting the full set of Raman active modes (including their symmetry classification), ${ }^{3}$ and two reporting the almost complete list, ${ }^{6,8}$ with a few missing peaks and minor disagreements with respect to each other. Five years ago, a computational study based on first-principle methods was published by some of the present authors, ${ }^{9}$ where the nature of the Raman active modes was discussed and the agreement with all the aforementioned experimental data resulted to be excellent concerning the position of the peaks.

However, to the best of our knowledge, the available information on the intensity of the peaks is still only qualitative. From the point of view of computer simulation, the calculation of Raman intensity is a highly-demanding task, and its implementation has been finalised only in very recent times. ${ }^{10,11}$ This paper reports the Raman intensities of grossular computed through this new scheme, and addresses a certain number of questions related to the few cases where important disagreements among the available experimental sets, and between the latter and the computed data set by Dovesi et al. ${ }^{9}$ were observed.

As a matter of facts, the quantitative prediction of Raman intensities is here shown to be important for understanding whether modes in the experimental spectra are missing or in disagreement with each other due to their low intensity, or because they are very close to a much larger peak, or for some other reasons (defects, impurities, low crystallinity, background).

Aside from the production of an accurate reference data set for the Raman spectrum of 
grossular, a second main purpose of this paper is showing that first-principle techniques are powerful tools able to predict with high accuracy the Raman features of complex minerals, such as garnets.

\section{COMPUTATIONAL METHODS}

All the calculations were performed by using an all electron Gaussian-type basis set and the hybrid B3LYP functional ${ }^{12-14}$ as implemented in the 2014 release of the CRYSTAL code. ${ }^{15,16}$ The approach here adopted has extensively been able to provide a highly accurate description of the IR vibrational properties of garnets (e.g. Ref. 17 and references therein).

Geometry optimization and calculation of vibrational frequencies were performed following the same schemes and adopting the same parameters, thresholds and basis set as described in a previous publication, ${ }^{9}$ where the Raman active vibrational frequencies of grossular were computed and analysed.

We rather focus here on the calculation of Raman intensities, as recently implemented in the CRYSTAL code. ${ }^{10}$ The relative intensities of Raman peaks were obtained through an analytical approach, which is an extension of the algorithms that allow for the calculation of infrared intensities. ${ }^{18}$ This formalism is based on combining gradients of mono- and bielectronic integrals ${ }^{19,20}$ with a Coupled Perturbed Hartree-Fock/Kohn Sham (CPHF/KS) scheme $^{21,22}$ for the response of the crystalline orbitals to a static electric field. Raman intensities for an oriented single crystal at a given temperature and laser wavelength are then computed according to Prosandeev et al. ${ }^{23}$

Further details on the methods can be found in Ref. ${ }^{15,16}$ Input and output files are available for download at http://www.theochem.unito.it/garnets/ .

\section{RESULTS AND DISCUSSION}

\section{A. Review of experimental data from the literature}

In the last twenty years, several groups have measured and characterized the Raman peaks of grossular. First, in 1991, Hofmeister and Chopelas ${ }^{3}$ classified the full set of 25 symmetry allowed modes. Some of the assignments were later reconsidered, in 2005, in a paper devoted to uvarovite, ${ }^{8}$ where a set of 24 wavenumbers is reported for grossular, showing 
some relevant differences. Pinet and $\mathrm{Smith}^{24}$ in 1993, while discussing solid solutions of the uvarovite-grossular-andradite series, reported measurements of an almost pure grossular end-member sample (there labeled G16); they assigned only 20 out of 25 peaks. A final major source of information is the work by Kolesov and Geiger ${ }^{6}$ published in 1998, which identified 22 modes.

Here and in the following we will refer to the four sets as Hof, ${ }^{3}$ Chop, ${ }^{8}$ Pinet, ${ }^{24}$ and Kol, ${ }^{6}$ for sake of brevity. Table I reports the wavenumbers of these fours sets, with the corresponding cross-statistics given in Table II. Overall, the four experimental datasets are in good agreement, the mean absolute difference being $7 \mathrm{~cm}^{-1}$ in the worst case. The set with relatively larger discrepancies is Pinet, showing 7 and $5 \mathrm{~cm}^{-1}$ when compared to Hof and Chop, respectively. The largest difference, $36 \mathrm{~cm}^{-1}$, is found when comparing Hof and Chop.

Table I shows that most of the modes were recognized nearly at the same wavenumber by all authors. However, it appears that certain modes cannot be unambiguously assigned to a given frequency:

1. the two lowest modes, 1 and 2, are characterized as a composite band by Hof and Pinet only; the corresponding frequencies are rather different, 178 and $185 \mathrm{~cm}^{-1}$, respectively. Chop identified mode 1 at a frequency much higher than all other authors, $214 \mathrm{~cm}^{-1}$ (in the paper itself the assignment is marked as doubtful);

2. mode $3\left(238-239 \mathrm{~cm}^{-1}\right)$ was not seen by Kol, and found at $248 \mathrm{~cm}^{-1}$ by Pinet;

3. mode 15 was characterized by Pinet as a composite band at the same wavelength as mode $14,513 \mathrm{~cm}^{-1}$. Other authors reported it at $526-529 \mathrm{~cm}^{-1}$;

4. mode 21, not seen by Kol, was assigned to $852-854 \mathrm{~cm}^{-1}$ by Hof and Pinet, and to $826 \mathrm{~cm}^{-1}$ by Chop. In fact, Hof and Pinet identified a composite band with mode 22, whereas Chop assumed a composite with mode 20;

5. mode 23, not seen by Kol and Pinet, has a $20 \mathrm{~cm}^{-1}$ difference between Hof and Chop assignments (904 and $884 \mathrm{~cm}^{-1}$, respectively).

Wavenumbers corresponding to these doubtful assignments are underlined in Table I. Notably, cross-statistics among the four experimental sets (Table II) show a relevant improve- 
ment if these doubtful frequencies are not taken into account: the largest mean absolute error decreases to $4 \mathrm{~cm}^{-1}$, the maximum absolute error to $7 \mathrm{~cm}^{-1}$.

\section{B. Simulated frequencies and intensities}

Computed Raman frequencies and intensities (both isotropic and directional) are reported in Table I, in comparison with the experimental frequencies. The mean absolute difference $|\overline{\Delta \nu}|$ between calculated and experimental frequencies is as small as $8,5,8$ and $8 \mathrm{~cm}^{-1}$ for Hof, Kol, Chop and Pinet, respectively; the maximum absolute deviation $|\Delta \nu|_{\max }$ is 32,13 , 37 and $31 \mathrm{~cm}^{-1}$. The set by Kol shows the best performance. Note that frequency values for many of the the doubtfully assigned modes (see Section III A above) show rather large discrepancies compared to our computed data. If doubtful frequencies are excluded from the statistics, the agreement for Hof, Chop and Pinet improves and gets closer to Kol (which has no doubtful assignments). Remaining discrepancies larger than $10 \mathrm{~cm}^{-1}$ are found in the ranges 520-640 $\mathrm{cm}^{-1}$ (modes 15, 16, 17 and 19, positive) and 810-890 $\mathrm{cm}^{-1}$ (modes 20, 22 and 23, negative).

A closer comparison between computed and experimental data helps in a proper characterization of the doubtful modes, with simulated intensities giving evidence to clarify their origin. Modes 1 and 2 are two distinct peaks with distance on the order of a few $\mathrm{cm}^{-1}$, as recognized by Kol, though their symmetry assignment of these two peaks is reversed compared to that from calculation. The almost null intensity of mode 1 is probably the main cause of its difficult identification. Apparently, the assignment by Hof and Pinet was made by simply merging mode 1 with the intense and neighboring mode 2 . Beside that, the origin of the large overestimation of mode 1 by Chop remains not clear. Mode 3 lies at the frequency proposed by Hof and Chop, with its low intensity probably being the reason for the missing value of Kol and the misattribution by Pinet. Mode 15 is independent from mode 14, as recognized by all but Pinet, and the peak shows a non-negligible intensity.

As regards mode 21, simulation indicates that Chop provides the right attribution, whereas Hof and Pinet put it in close correspondence with mode 22, which is about $25 \mathrm{~cm}^{-1}$ higher in frequency, and Kol could not identify it. Once again, the almost null computed intensity substantiates these experimental difficulties. Aslo mode 23 was correctly assigned only by Chop, whereas Kol and Pinet did not find it, and Hof listed it $20 \mathrm{~cm}^{-1}$ higher. In 
this case, the computed intensity results high enough and non-negligible. However, mode 24 (the most intense in the whole spectrum) has a computed frequency only $4 \mathrm{~cm}^{-1}$ higher, which can cause peak overlapping. Actually, modes 23 and 24 are characterized by different symmetry ( $\mathrm{E}_{g}$ and $\mathrm{A}_{g}$, respectively), so that directional measurements could have revealed it. In fact, Figure 2 shows that contamination among symmetries is occurring, with the leakage from $\mathrm{A}_{g}$ symmetry hiding the $\mathrm{E}_{g}$ peak (see Section III C below for further discussion on Figure 2).

Finally, three more modes, 8, 13 and 17, show very small computed intensities. Apart from the set by Pinet, where modes 8 and 17 were not identified, experimental assignments are in quite good agreement. This good outcome can be related to the isolation characterizing these peaks: closest modes lie at a distance of at least $10 \mathrm{~cm}^{-1}$.

\section{Polycrystalline and single crystal spectra}

Figure 1 compares the simulated and experimental Raman spectrum for a polycrystalline sample. As powder experimental spectra relative to the data sets of Table I were not available in digitized form, we will refer here to a spectrum obtained from the Lyon Raman database. $^{25}$ Despite the several other experiments available in the RUFF database ${ }^{26}$ do not differ significantly from that from Lyon Raman database, the latter looks of higher quality.

The overall comparison between simulated and experimental spectra is very good. In particular, the ranking for the four most intense peaks is the same: $\sim 880 \mathrm{~cm}^{-1}, \sim 370 \mathrm{~cm}^{-1}$, $\sim 550-560 \mathrm{~cm}^{-1}, \sim 820-830 \mathrm{~cm}^{-1}$. Minor discrepancies are seen in the intensity of the peaks around 180 and $510 \mathrm{~cm}^{-1}$, in the frequency of the peaks at $\sim 550-560 \mathrm{~cm}^{-1}$ and $\sim 820-830$ $\mathrm{cm}^{-1}$, in both frequency and intensity of the peak at $\sim 630-640 \mathrm{~cm}^{-1}$.

In Figure 2 our simulated directional spectra are compared with those by $\mathrm{Kol}^{6}$. The agreement is satisfactory also in this case, despite three major intensity overestimation in the case of $\mathrm{F}_{2 g}$ symmetry $\left(\sim 510,630-640\right.$ and $\left.1005 \mathrm{~cm}^{-1}\right)$. However, in this case the most relevant aspect is how simulation can help in distinguishing fundamental mode peaks from leakages. The latter are marked in Figure 2 by dotted lines. Note how, in the region 200-350 $\mathrm{cm}^{-1}$ of the $\mathrm{E}_{g}$ spectrum, at least three peaks of comparable intensity are experimentally observed. Thanks to our simulation, we can unambiguously state that two of them are leakages from the $\mathrm{F}_{2 g}$ symmetry. It is also easy to recognize leakage of two $\mathrm{F}_{2 g}$ and one 
$\mathrm{A}_{g}$ modes in the range $800-900 \mathrm{~cm}^{-1}$ of the $\mathrm{E}_{g}$ spectrum. Finally, in the $\mathrm{F}_{2 g}$ spectrum the leakage of $\mathrm{A}_{g}$ most intense mode is quite evident around $880 \mathrm{~cm}^{-1}$.

\section{CONCLUSIONS}

By means of a full $a b$ initio approach, we have simulated the Raman spectrum of grossular garnet. The CRYSTAL program has been used, adopting a Gaussian basis set and a hybrid functional such as B3LYP.

Agreement with the experimental data is excellent, and has allowed us to solve the remaining controversies among experimental groups concerning the assignment of Raman peaks. Thanks to the availability of computed intensities, we have been able to show that some vibrational modes of grossular, though Raman active according to symmetry considerations, have nearly zero intensity, and in fact cannot be seen in the experimental spectrum. Misattributions due to leakages have been properly addressed, too.

This study confirms the reliability of modern quantum chemical methods in the simulation of vibrational spectra of materials, highlighting their relevant role in the interpretation of experimental data.

\section{ACKNOWLEDGEMENTS}

This research was supported by Curtin University through the Curtin Research Fellowship scheme. iVEC@Murdoch and the Australian National Computational Infrastructure facilities are also acknowledged for the provision of computer time. 
Figures 


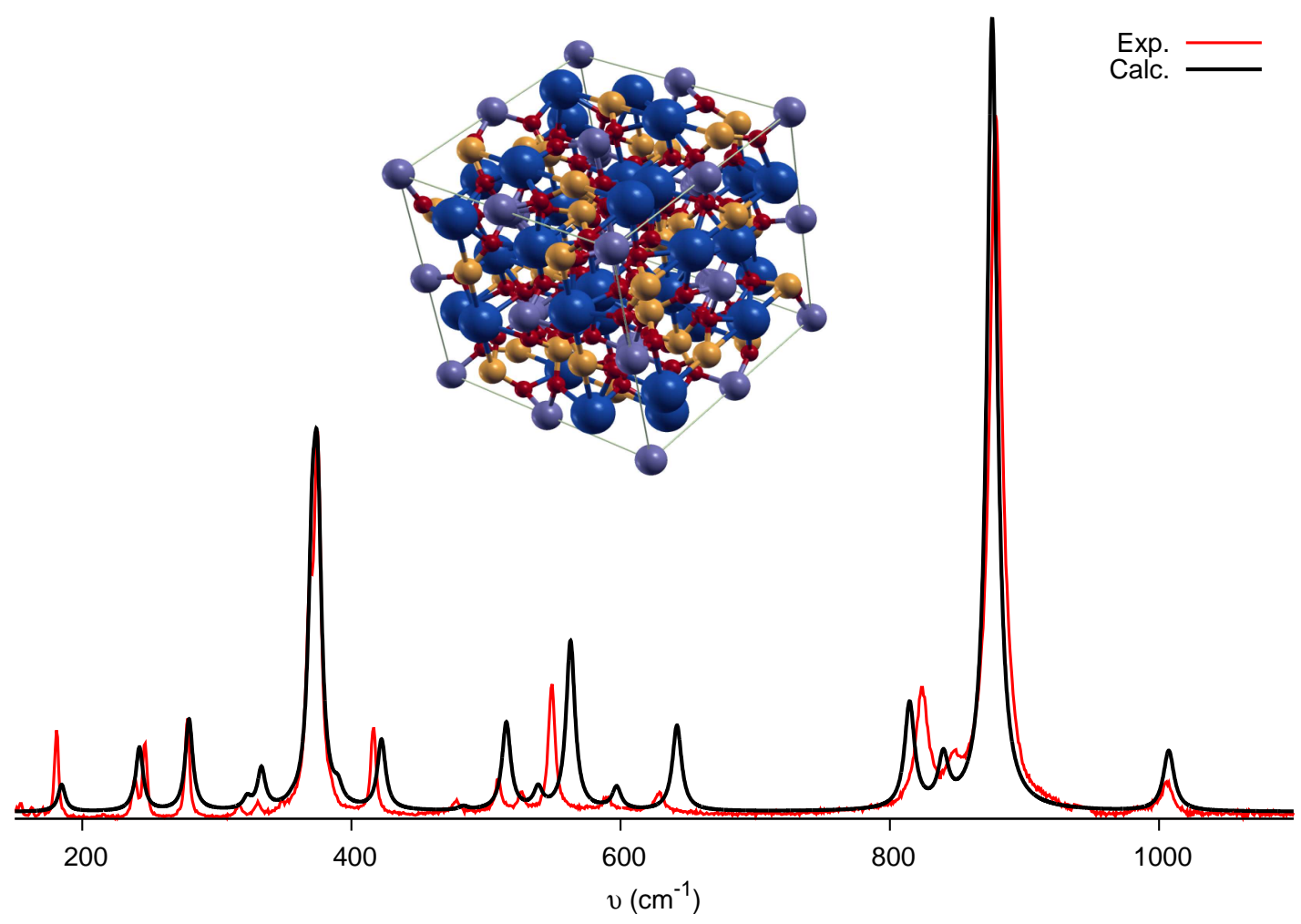

FIG. 1: Calculated and experimental ${ }^{25}$ polycristalline (powder) Raman spectra of grossular; wavenumbers range from 100 to $1400 \mathrm{~cm}^{-1}$. Both spectra are normalized to the most intense peak. Experimental conditions are $300 \mathrm{~K}$ and $514.5 \mathrm{~nm}$ for temperature and laser frequency, respectively. The baseline was subtracted from the experimental data. The simulated spectrum is obtained through Lorentzian broadening of the peaks; a non uniform broadening parameter was adopted, that varies linearly from 5 to $3 \mathrm{~cm}^{-1}$ when going from 100 to $1100 \mathrm{~cm}^{-1}$, respectively; this strategy improves the fit to the experiment, as already pointed out in the case of jadeite. ${ }^{27}$ Note that peak positions are reported exactly as computed, with no further processing (i.e. down shift). A representation of the crystal unit cell is also shown. 


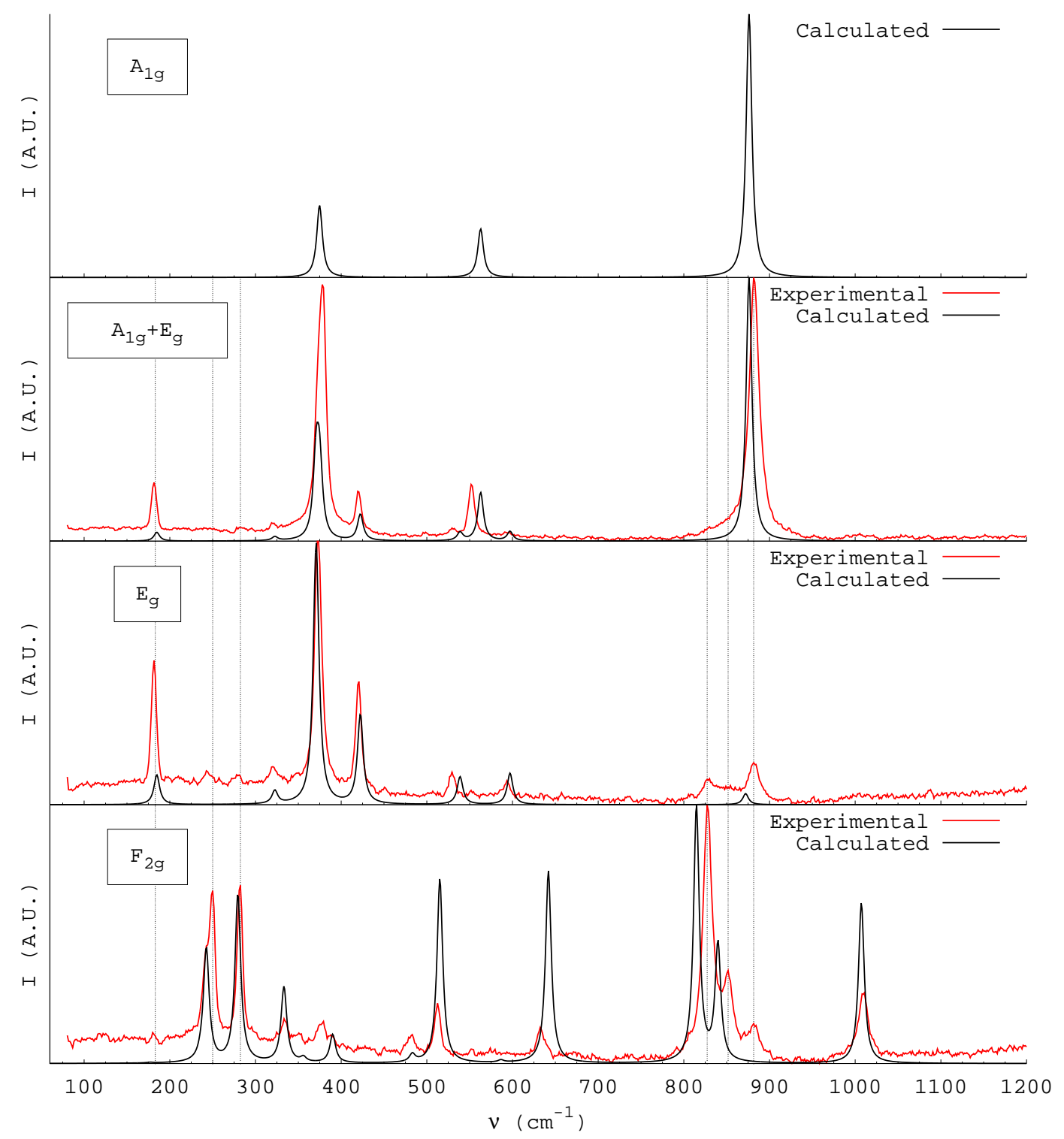

FIG. 2: Calculated and experimental ${ }^{6}$ single crystal Raman spectra of grossular for $A_{1 g}+E_{g}$, $E_{g}$ and $F_{2 g}$ mode symmetries. Wavenumbers range from 0 to $1200 \mathrm{~cm}^{-1}$. In each panel spectra are normalized to the most intense peak. Experimental conditions are $300 \mathrm{~K}$ and $488 \mathrm{~nm}$ for temperature and laser frequency, respectively. See caption to Figure 1 for more details on the construction of the spectra. Dotted lines are reported as a guide for the indentification of peak leakages, as discussed in the text. 
Tables 


\begin{tabular}{|c|c|c|c|c|c|c|c|c|c|c|c|c|}
\hline \multirow[b]{3}{*}{ Mode } & \multirow[b]{3}{*}{ Symm. } & \multirow{2}{*}{\multicolumn{3}{|c|}{$\begin{array}{c}\text { Calculated } \\
\text { This work }\end{array}$}} & \multicolumn{8}{|c|}{ Experimental } \\
\hline & & & & & \multicolumn{2}{|c|}{ Hof $^{3}$} & \multicolumn{2}{|c|}{$\mathrm{Kol}^{6}$} & \multicolumn{2}{|c|}{ Chop $^{8}$} & \multicolumn{2}{|c|}{ Pinet $^{24}$} \\
\hline & & $\nu$ & $I_{\text {iso }}$ & $I_{\text {dir }}$ & & $\Delta \nu$ & & $\Delta \nu$ & & $\Delta \nu$ & $\nu$ & $\Delta \nu$ \\
\hline 1 & $F_{2 g}$ & 176.8 & 0.2 & 0.2 & $\underline{178}$ & -1 & 186 & -9 & $\underline{214}$ & -37 & $\underline{185}$ & -8 \\
\hline 2 & $E_{g}$ & 184.8 & 23.4 & 33.4 & 178 & 7 & 181 & 4 & 178 & 7 & 185 & 0 \\
\hline 3 & $F_{2 g}$ & 236.8 & 1.6 & 1.1 & 238 & -1 & - & - & 239 & -2 & $\underline{248}$ & -11 \\
\hline 4 & $F_{2 g}$ & 242.6 & 57.2 & 40.9 & 246 & -3 & 247 & -4 & 246 & -3 & 250 & -7 \\
\hline 5 & $F_{2 g}$ & 279.4 & 84.8 & 60.6 & 278 & 1 & 280 & -1 & 278 & 1 & 283 & -4 \\
\hline 6 & $E_{g}$ & 322.6 & 10.2 & 14.6 & 317 & 6 & 320 & 3 & 316 & 7 & 318 & 5 \\
\hline 7 & $F_{2 g}$ & 333.2 & 38.3 & 27.3 & 330 & 3 & 333 & 0 & 330 & 3 & 334 & -1 \\
\hline 8 & $F_{2 g}$ & 355.9 & 2.3 & 1.7 & 349 & 7 & 351 & 5 & 348 & 8 & - & - \\
\hline 9 & $E_{g}$ & 370.8 & 205.8 & 294.0 & 369 & 2 & 373 & -2 & 368 & 8 & - & - \\
\hline 10 & $A_{g}$ & 374.8 & 273.5 & 273.5 & 374 & 1 & 376 & -1 & 374 & 1 & 378 & -3 \\
\hline 11 & $F_{2 g}$ & 390.1 & 14.3 & 10.2 & 383 & 7 & 389 & 1 & - & - & - & - \\
\hline 12 & $E_{g}$ & 422.4 & 70.0 & 100.1 & 416 & 6 & 420 & 2 & 416 & 6 & 420 & 2 \\
\hline 13 & $F_{2 g}$ & 483.1 & 3.8 & 2.8 & 478 & 5 & 483 & 0 & 478 & 5 & 482 & 1 \\
\hline 14 & $F_{2 g}$ & 515.2 & 93.0 & 66.4 & 509 & 6 & 512 & 3 & 508 & 7 & 513 & 2 \\
\hline 15 & $E_{g}$ & 538.8 & 21.8 & 31.2 & 526 & 13 & 529 & 10 & 526 & 13 & $\underline{513}$ & 26 \\
\hline 16 & $A_{g}$ & 562.8 & 184.2 & 184.2 & 549 & 14 & 550 & 13 & 549 & 14 & 552 & 11 \\
\hline 17 & $F_{2 g}$ & 586.5 & 1.1 & 0.8 & 577 & 10 & 582 & 5 & 577 & 10 & - & - \\
\hline 18 & $E_{g}$ & 597.1 & 24.6 & 35.2 & 590 & 7 & 592 & 5 & 590 & 7 & 594 & 3 \\
\hline 19 & $F_{2 g}$ & 641.9 & 96.9 & 69.2 & 629 & 13 & 630 & 12 & 629 & 13 & 635 & 7 \\
\hline 20 & $F_{2 g}$ & 814.7 & 129.1 & 92.2 & 826 & -11 & 827 & -12 & 827 & -12 & 828 & -13 \\
\hline 21 & $E_{g}$ & 823.1 & 0.2 & 0.3 & $\underline{852}$ & -29 & - & - & 826 & -3 & $\underline{854}$ & -31 \\
\hline 22 & $F_{2 g}$ & 839.8 & 59.1 & 42.2 & 850 & -10 & 848 & -8 & 851 & -11 & 854 & -14 \\
\hline 23 & $E_{g}$ & 872.1 & 8.7 & 12.4 & $\underline{904}$ & -32 & - & - & 884 & -12 & - & - \\
\hline 24 & $A_{g}$ & 876.1 & 1000.0 & 1000.0 & 881 & -5 & 880 & -4 & 882 & -6 & 884 & -8 \\
\hline 25 & $F_{2 g}$ & 1007.2 & 80.9 & 57.8 & 1007 & 0 & 1007 & 0 & 1007 & 0 & 1014 & $\begin{array}{ll}4 & -7 \\
\end{array}$ \\
\hline $\mathrm{N}$ & & & & & 25 & (22) & & 22 & 24 & (23) & 20 & (16) \\
\hline$\overline{\Delta \nu}$ & & & & & 1 & & & 1 & & (2) & -3 & $(-2)$ \\
\hline$|\overline{\Delta \nu}|$ & & & & & 8 & & & 5 & & (7) & & (6) \\
\hline$|\Delta \nu|_{\max }$ & & & & & 32 & & & 13 & 37 & (14) & 31 & (14) \\
\hline
\end{tabular}


TABLE I: Calculated and experimental Raman properties of grossular. Frequencies $\nu$ are in $\mathrm{cm}^{-1}$. Calculated Raman intensities $I$ are normalized to the most intense peak, whose value is arbitrarily set to 1000.0; they refer to the experimental conditions $T=293 \mathrm{~K}, \lambda=488 \mathrm{~nm}$; both powder (polycristalline) and single crystal intensities are reported $\left(\mathrm{I}_{i s o}\right.$ and $\mathrm{I}_{d i r}$, respectively). Underlined font mark modes with doubtful attribution (1, 21 and 23 for Hof; 1 for Chop; 1, 3, 15 and 21 for Pinet). Differences $\Delta \nu$ are equal to $\nu_{\text {calc }}-\nu_{\exp } . N$ is the number of peaks considered in each statistics; $\overline{\Delta \nu}, \overline{|\Delta \nu|}$ and $|\Delta \nu|_{\max }$ are the mean difference, the mean absolute difference and the absolute maximum difference evaluated over the set of $N$ peaks. Numbers in parentheses refer to the statistics obtained when excluding modes with doubtful attribution. 


\begin{tabular}{|c|c|c|c|c|}
\hline & & $\mathrm{Kol}^{6}$ & Chop $^{8}$ & Pinet $^{2}$ \\
\hline \multirow{4}{*}{$\mathrm{Hof}^{3}$} & $\mathcal{N}$ & $22(21)$ & $24(21)$ & $20(16)$ \\
\hline & $\overline{\Delta \nu}$ & $-3(-2)$ & $0(0)$ & $-4(-4)$ \\
\hline & $|\overline{\Delta \nu}|$ & $3(3)$ & $4(0)$ & $5(4)$ \\
\hline & $|\Delta \nu|_{\max }$ & $8(6)$ & $36(1)$ & $13(7)$ \\
\hline \multirow{4}{*}{$\mathrm{Kol}^{6}$} & $\mathcal{N}$ & & $21(20)$ & $18(16)$ \\
\hline & $\overline{\Delta \nu}$ & & $1(2)$ & $-1(-2)$ \\
\hline & $|\overline{\Delta \nu}|$ & & $4(3)$ & $3(3)$ \\
\hline & $|\Delta \nu|_{\max }$ & & $28(5)$ & $16(7)$ \\
\hline \multirow{4}{*}{ Chop $^{8}$} & $\mathcal{N}$ & & & $20(16)$ \\
\hline & $\overline{\Delta \nu}$ & & & $-3(-4)$ \\
\hline & $|\overline{\Delta \nu}|$ & & & $7(4)$ \\
\hline & $|\Delta \nu|_{\max }$ & & & $29(7)$ \\
\hline
\end{tabular}

TABLE II: Crossed statistical analysis among experimental data. $\mathcal{N}$ is the number of modes considered in each statistics. Numbers in parentheses refer to the statistics obtained when excluding modes with doubtful attribution. For further details see caption to Table I. 
1 Geiger CA. Garnet: A Key Phase in Nature, the Laboratory, and Technology. Elements 2013, $9,447-452$.

2 Baxter EF, Caddick MJ, Ague JJ. Garnet: Common Mineral, Uncommonly Useful. Elements 2013, 9, 415-419.

3 Hofmeister AM, Chopelas A. Vibrational spectroscopy of end-member silicate garnets. Phys. Chem. Miner. 1991 17, 503-526.

${ }^{4}$ Hofmeister AM, Fagan TJ, Campbell KM, Schaal RB. Single-crystal IR spectroscopy of PyropeAlmandine garnets with minor amounts of Mn and Ca. Am. Mineral. 1996, 81, 418-428.

5 McAloon BP, Hofmeister AM. Single-crystal IR spectroscopy of grossular-andradite garnets. Am. Mineral. 1995, 80, 1145-1156.

6 Kolesov B, Geiger CA. Raman Spectra of Silicate Garnets. Phys. Chem. Miner. 1998, 25, $142-151$.

7 Kolesov B, Geiger CA. Low-temperature single-crystal Raman spectrum of pyrope. Phys. Chem. Miner. 2000, 27, 645-649.

8 Chopelas A. Single crystal Raman spectrum of uvarovite, $\mathrm{Ca}_{3} \mathrm{Cr}_{2} \mathrm{Si}_{3} \mathrm{O}_{12}$. Phys. Chem. Miner. $2005,32,525-530$.

9 Dovesi R, Valenzano L, Pascale F, Zicovich-Wilson C, Orlando R. Ab initio quantum-mechanical simulation of the Raman spectrum of grossular. J. Raman Spectrosc. 2009, 40, 416-418.

10 Maschio L, Kirtman B, Rérat M, Orlando R, Dovesi R. Ab initio analytical Raman intensities for periodic systems through a coupled perturbed Hartree-Fock/Kohn-Sham method in an atomic orbital basis. I. Theory. J. Chem. Phys. 2013, 139, 164101.

11 Maschio L, Kirtman B, Rérat M, Orlando R, Dovesi R. Ab initio analytical Raman intensities for periodic systems through a coupled perturbed Hartree-Fock/Kohn-Sham method in an atomic orbital basis. II. Validation and comparison with experiments. J. Chem. Phys. 2013, 139,164102 .

12 Becke AD. Density-functional thermochemistry .3. The role of exact exchange. J. Chem. Phys. 1993, $98,5648-5652$.

13 Lee C, Yang W, Parr RG. Development of the Colle-Salvetti correlation-energy formula into a functional of the electron density. Phys. Rev. B 1988, 37, 785-789. 
14 Koch W, Holthausen MC. A Chemist's Guide to Density Functional Theory. Wiley-VCH Verlag GmbH: Weinheim, 2000.

15 Dovesi R, Saunders VR, Roetti C, Orlando R, Zicovich-Wilson CM, Pascale F, Civalleri B, Doll K, Harrison NM, Bush IJ, et al.. CRYSTAL14 User's Manual. Università di Torino, Torino 2014.

16 Dovesi R, Orlando R, Erba A, Zicovich-Wilson C, Civalleri B, Casassa S, Maschio L, Ferrabone M, De La Pierre M, D'Arco P, et al.. CRYSTAL14: A Program for the Ab Initio Investigation of Crystalline Solids. Int. J. Quantum Chem. submitted on 15/1/2014; .

17 Dovesi R, De La Pierre M, Ferrari AM, Pascale F, Maschio L, Zicovich-Wilson CM. The IR vibrational properties of six members of the garnet family: A quantum mechanical $a b$ initio study. Am. Mineral. 2011, 96, 1787-1798.

18 Maschio L, Kirtman B, Orlando R, Rérat M. Ab initio analytical infrared intensities for periodic systems through a coupled perturbed Hartree-Fock/Kohn-Sham method. J. Chem. Phys. 2012, 137, 204113.

19 Doll K, Harrison NM, Saunders VR. Analytical Hartree-Fock gradients for periodic systems. Int. J. Quantum. Chem. 2001, 82, 1-13.

20 Doll K. Implementation of analytical Hartree-Fock gradients for periodic systems. Comp. Phys. Comm. 2001 137, 74-88.

21 Ferrero M, Rérat M, Kirtman B, Dovesi R. Calculation of first and second static hyperpolarizabilities of one- to three-dimensional periodic compounds. Implementation in the CRYSTAL code. J. Chem. Phys. 2008, 129, 244110.

22 Ferrero M, Rérat M, Orlando R, Dovesi R. The calculation of static polarizabilities of 1-3D periodic compounds. The implementation in the CRYSTAL code . J. Comput. Chem. 2008, 29, $1450-1459$.

23 Prosandeev S, Waghmare U, Levin I, Maslar J. First-order Raman spectra of $\mathrm{AB}^{\prime}{ }_{1 / 2} \mathrm{~B}^{\prime \prime}{ }_{1 / 2} \mathrm{O}_{3}$ double perovskites. Phys. Rev. B 2005, 71, 214307.

24 Pinet M, Smith D. Raman microspectrometry of garnets $\mathrm{X}_{3} \mathrm{Y}_{2} \mathrm{Z}_{3} \mathrm{O}_{12}$ : I. The natural calcic series uvarovite-grossular-andradite. Schweiz. Mineral. Petrogr. Mitt. 1993, 73, 21-40.

25 http://www.ens-lyon.fr/LST/Raman/.

26 http://rruff.info/grossular/.

27 Prencipe M, Maschio L, Kirtman B, Salustro S, Erba A, Dovesi R. Raman Spectrum of 
$\mathrm{NaAlSi}_{2} \mathrm{O}_{6}$ Jadeite. A Quantum Mechanical Simulation. submitted to J. Phys. Chem. A 2014; . 